INSERM, AP-HP, and the Fondation FondaMental (http://www. fondation-fondamental.org).

This project was approved by the CNS Institutional Review Board at the National Institutes of Health and by the GAIN Data
Access Committee. Informed consent was obtained from all subjects.

Additional acknowledgements are included at http://mapgenetics. nimh.nih.gov.

\title{
CYP2D6 ultrarapid metabolism and early dropout from fluoxetine or amitriptyline monotherapy treatment in major depressive patients
}

EM Peñas-LLedó, HD Trejo, P Dorado, A Ortega, H Jung, E Alonso, MEG Naranjo, M López-López and A LLerena

Molecular Psychiatry (2013) 18, 266; doi:10.1038/mp.2012.108; published online 4 December 2012

Corrections to: Molecular Psychiatry advance online publication, 26 June 2012; doi: 10.1038/mp.2012.91

Following the online publication of this article, the authors noted an error in the table: below 'yes' (with the number of individuals who discontinued from ADP treatment between parentheses), the word 'no' should appear instead of 'number'.

\section{De novo induction of amyloid- $\beta$ deposition in vivo}

R Morales, C Duran-Aniotz, J Castilla, LD Estrada and C Soto

Molecular Psychiatry (2013) 18, 266; doi:10.1038/mp.2012.153; published online 4 December 2012

Corrections to: Mol Psychiatry advance online publication, 4 October 2011; doi: 10.1038/mp.2011.120

The authors would like to correct a labeling mistake in Figure 4 of the article. The top two panels in the left (developed with anti-A $\beta$ antibody) correspond to an animal killed at 450 days post inoculation and not 585 days as stated in the labeling. Indeed, the panel shown at $\times 200$ magnification corresponds to a new picture taken with higher resolution of the same section shown in
Figure 3a (center panel). The other two top panels in the right (stained with thioflavin $\mathrm{S}$ and anti-GFAP antibody, respectively) are correctly labeled as coming from an animal killed at 585 days post inoculation. This mistake does not affect in any way the results or conclusions of the experiment, as the purpose of Figure 4 was to show a morphological and staining comparison of representative deposits observed in HuAPPwt transgenic mice after inoculation with Alzheimer's brain homogenate and those developed spontaneously in a mice expressing human mutant amyloid precursor protein (Tg2576). 